\title{
Assessment of the Correlation Between Carotid Artery Plaque Density Determined by Histogram Analysis and Positive Remodeling on Computerized Tomography Angiography Masaki Ogawa, ${ }^{1, *}$ Yoshiyuki Ozawa, ${ }^{1}$ Masahiro Muto, ${ }^{1}$ Hiroyuki Katano, ${ }^{2}$ Kazuo Yamada, ${ }^{2}$ Toshiyasu Miura, ${ }^{2}$ Noriyuki Matsukawa, ${ }^{3}$ and Yuta Shibamoto ${ }^{1}$ \\ ${ }^{1}$ Department of Radiology, Nagoya City University Graduate School of Medical Sciences, Nagoya, Japan \\ ${ }^{2}$ Department of Neurosurgery, Nagoya City University Graduate School of Medical Sciences, Nagoya, Japan \\ ${ }^{3}$ Department of Neurology, Nagoya City University Graduate School of Medical Sciences, Nagoya, Japan \\ "Corresponding author: Masaki Ogawa, Department of Radiology, Nagoya City University Graduate School of Medical Sciences, Kawasumi, Mizuho-cho, Mizuho-ku, Nagoya, Japan. Tel: +81-528538276, Fax: +81-528525244, E-mail: eternal@fj9.so-net.ne.jp
}

Received 2016 November 07; Revised 2017 April 24; Accepted 2017 May 08.

\begin{abstract}
Background: CT angiography (CTA) was routinely performed for carotid artery disease, but the plaque characterization based on measurement of density may be inaccurate, influenced by adjacent calcification and contrast agent. Remodeling ratio was one of the additional new objective indexes for plaque characterization. On coronary CTA, the utility of histogram analysis was reported in a few studies.

Objectives: The purpose of this study was to evaluate the diagnostic value of histogram analysis of carotid artery plaque density and positive remodeling on CTA for the diagnosis of pathologically proven complicated carotid artery plaques.

Patients and Methods: We retrospectively evaluated 29 patients with atherosclerotic carotid artery stenosis treated by carotid endarterectomy. On CTA, the boundaries of non-calcified plaques were manually traced at the site of the greatest stenosis. In addition to the mean plaque density, the percentage of pixels (PP) with a density of less than 30 Hounsfield units $(\mathrm{HU})(\mathrm{PP}<30)$ was calculated using the histogram analysis. We calculated the remodeling ratio by dividing the cross-sectional artery area at the greatest stenosis by normal reference-segment artery area. These results of CTA analysis were compared with the results of histological analysis based on the American heart association criteria.

Results: Histologically, 13 of the 29 patients had complicated plaques. $\mathrm{PP}<30$ and the remodeling ratio were higher in patients with complicated plaques than in those with uncomplicated plaques $(\mathrm{P}=0.023$ and 0.002 , respectively). Whereas, the mean plaque density did not differ between the two groups $(\mathrm{P}=0.062)$. Correlation between $\mathrm{PP}<30$ and the remodeling ratio was stronger than the correlation between $\mathrm{PP}<30$ and the mean density (Spearman's rs $=0.76$ vs. 0.65 , respectively).

Conclusion: Histogram analysis of the carotid artery plaque density may be more useful for the evaluation of atherosclerosis on CTA compared with the conventional analysis of plaque densities, and the combined analysis of histogram analysis and the remodeling ratio may help to predict future stroke events.
\end{abstract}

Keywords: Atherosclerotic Carotid Artery Plaque, CT Angiography, Histogram Analysis, Positive Remodeling

\section{Background}

Atherosclerotic disease of the extracranial carotid arteries is an important cause of stroke. Carotid endarterectomy (CEA) or stent placement is considered to prevent stroke for patients with symptomatic or asymptomatic carotid stenosis above a certain degree $(1,2)$. However, patients with mild stenosis in the internal carotid artery (ICA) can unexpectedly experience strokes. Vulnerable atherosclerotic plaques have been reported to be an additional risk of atherosclerotic disease (2-4). The vulnerability to rupture of atherosclerotic plaques is related to the intrinsic composition, such as the hemorrhage of intraplaque and lipid core size (2-6).

The coronary artery responds to the growth of atherosclerotic plaques in different ways: by shrinkage of vessel wall (negative remodeling) or by outward expansion (positive remodeling) (7). Plaques showing positive remodeling tended to show higher macrophage count and have large lipid core compared to those without positive remodeling (3). Positive remodeling was previously thought to be a delayed response of luminal stenosis during the progression of atherosclerosis (3), but it was 
recently presumed to be associated with plaque inflammation and increased tendency for rupture $(3,5,8)$. For subjects with severe carotid artery stenosis, greater positive remodeling was observed in patients with cerebral ischemic symptoms than in patients with no symptoms in the previous study $(3,5)$. Plaque characterization or positive remodeling provides accurate risk stratification for future cerebral ischemic events.

Several imaging techniques and methods have demonstrated the ability to predict cerebral ischemic events; however, there are no definite criteria and we need to evaluate several factors as a whole. Multi-detector computed tomography (MDCT) allows evaluation of the carotid artery with thin slice thickness (no more than $1 \mathrm{~mm}$ ), and more detailed analysis of carotid artery stenosis and plaque components. Previous studies reported that differences in Hounsfield unit (HU) on MDCT can characterize components of atherosclerotic plaque $(2,4,9)$. However, high density influenced by luminal enhancement and calcification of the plaque may limit calculation of the accurate mean plaque density, leading to inaccurate characterization of fibrous and lipid-rich plaques $(10,11)$. For the coronary atherosclerotic plaque characterization, a few studies have reported the potential role of a quantitative histogram analysis for distribution of pixels showing low density $(10,11)$. Marwan et al. (11) reported the utility of histogram analysis for coronary artery plaque characterization on CT angiography (CTA): the distribution of pixels with density $<30 \mathrm{HU}$ calculated by histogram analysis may contribute to more reliable plaque classification. As in the coronary arteries, the extent of positive remodeling of the ICA may indicate underlying atherosclerotic plaque vulnerability and ischemic symptoms $(3,5,8)$. Therefore, both histogram analysis of plaque density and positive remodeling on CTA may contribute to carotid plaque characterization and assessment of the risk of stroke.

\section{Objectives}

The purpose of this study was to evaluate the diagnostic value of histogram analysis of carotid artery plaque density and positive remodeling for the diagnosis of pathologically proven complicated carotid artery plaques.

\section{Patients and Methods}

\subsection{Patients}

This was a retrospective study approved by medical research ethics committee of our institution. The requirement for informed consent was waived. The privacy of the patients was fully protected. Between January 2009 and February 2014, we selected the patients following search of electronic medical records in our institution. We applied the following inclusion criteria to patients: acquisition of carotid CTA on the same CT scanner and imaging parameters within 3 months before CEA following the previous study (2), and treatment of atherosclerotic carotid artery stenosis by CEA. An exclusion criterion was the insufficient quality of CTA due to artifacts or poor visualization of the artery. One carotid artery was excluded from the study because of poor visualization of ICA due to near occlusion. Twenty-nine patients (mean age: $69 \pm 5$ years) with a total of 29 lesions were finally enrolled in this study. Characteristics of the patients are summarized in Table 1 . Clinical information of all patients was obtained from the electronic medical records at our institution including other clinical findings.

\subsection{CT Examination}

Carotid CTA was performed by a 64-slice dual source CT scanner (SOMATOM Definition; Siemens Medical Solutions, Forchheim, Germany). The imaging acquisition parameters for CTA were as follows: spiral mode 0.5-second gantry rotation, $32 \times 0.6 \mathrm{~mm}$ collimation, $0.9-1.5 \mathrm{pitch}$ factor, $1.0 \mathrm{~mm}$ section thickness, $0.5-0.8 \mathrm{~mm}$ reconstruction interval, $120 \mathrm{kVp}$ tube voltage, a tube current of 400 - 650 mAs, and kernel B30-31f. $50 \mathrm{~mL}$ of iodine contrast medium, iopamidol (Iopamiron 300; Bayer Schering Pharma, Berlin, Germany) or iohexol (Omnipaque 300; Dainichi Sankyo, Tokyo, Japan), was injected and followed by $20 \mathrm{~mL}$ of a saline chaser at a flow rate of $4.0 \mathrm{~mL} / \mathrm{s}$. An automated bolustiming program determined optimal timing of CTA acquisition. Images were obtained from the level of the inferior orbits to the aortic arch.

\subsection{Image Data Analysis}

CT images were analyzed by one neuroradiologist (M.O.) who was blinded to the clinical and pathological information. Multiplanar reconstruction (MPR) was performed to produce two-dimensional images showing the cross-sectional profile of a vessel along its length on a computer workstation (Aquarious iNtuition, Version 4.4.7; TeraRecon, Inc, San Mateo, CA, USA). Using a window level of 250 and window width of 700, the region of maximal luminal stenosis was visually identified on MPR and axial images on the workstation (Figure $1 \mathrm{~A}$ and $\mathrm{B}$ ). The degree of stenosis was determined following the criteria of the North American symptomatic carotid endarterectomy trial (NASCET). The outer vessel contouring was manually performed to calculate the cross-sectional vessel area 
Table 1. Comparison of Clinical Characteristics Between Type-VI and Non-Type-VI Groups ${ }^{\mathrm{a}}$

\begin{tabular}{|c|c|c|c|c|}
\hline Variables & All & Type-VI & Non-Type-VI & P Value \\
\hline Number of patients & 29 & 13 & 16 & \\
\hline Age, $y$ & $69 \pm 5$ & $68 \pm 5$ & $70 \pm 5$ & 0.37 \\
\hline Male Gender & $24(83)$ & $12(92)$ & $12(75)$ & 0.23 \\
\hline Symptoms & $25(86)$ & $11(85)$ & $14(88)$ & 0.83 \\
\hline Hypertension & $25(83)$ & $11(85)$ & $14(88)$ & 0.83 \\
\hline Diabetes & $9(31)$ & $4(31)$ & $5(31)$ & 0.98 \\
\hline Smoking & $22(76)$ & $11(85)$ & $11(69)$ & 0.33 \\
\hline Dyslipidemia & $16(55)$ & $9(69)$ & $7(44)$ & 0.18 \\
\hline Coronary artery disease & $5(17)$ & $1(8)$ & $4(25)$ & 0.23 \\
\hline Stenosis (NASCET) & $76.1 \pm 13.9$ & $73.9 \pm 16.9$ & $77.9 \pm 11.1$ & 0.61 \\
\hline
\end{tabular}

Abbreviation: NASCET, north american symptomatic carotid endarterectomy trial.

${ }^{\mathrm{a}}$ Values are expressed as Mean \pm SD or No (\%).

(CSA) on slices of maximum stenosis, reference CSA calculated at the nearest distal segments without atherosclerotic plaques (Figure $1 \mathrm{~B}$ and $\mathrm{C}$ ). According to previously reported methods $(3,5)$, the remodeling ratio was calculated as follows:

Remodeling ratio $=($ CSA at the point of maximum stenosis / reference CSA at the distal portion)

On the Image J program (Image J $1.48 \mathrm{v}$; national institutes of health, MD, USA), non-calcified plaques in cross sections at the point of maximum stenosis were manually traced at a short distance from calcification, vessel lumen, or wall to avoid partial volume effect, and histograms of the CT value distribution were obtained. Graphs were created using Excel (Microsoft, Redmond, Washington) (Figure 2). Based on the previous coronary CTA histogram study using a similar CT scanner (11), the percentage of pixels with density $<30 \mathrm{HU}(\mathrm{PP}<30)$ and mean density of the carotid plaques were calculated.

We selected $30 \mathrm{HU}$ as the cutoff for low-density plaques for the following reason. In the coronary CTA histogram analysis, Schlett et al. (10) and Marwan et al. (11) reported 60 and $30 \mathrm{HU}$ as the cutoff, respectively. In a previous metaanalysis study, significant differences were found for the mean HU criteria of atherosclerotic plaques according to the CT vendor and number of detector rows with no correlation to the lumen contrast enhancement (12). Also, the HU criteria of carotid lipid-rich plaques may be lower than those of coronary arteries, caused by less partial volume effect or movement (12). Therefore, we selected $30 \mathrm{HU}$ as the cutoff because Marwan et al. (11) used a CT scanner (SOMATOM Definition) similar to ours in their coronary CTA study.

\subsection{Histological Grading of Carotid Artery Plaques Obtained by} CEA

All lesion samples obtained by CEA were used to assess and categorize the histological classification of atherosclerotic plaques. The CEA specimens were fixed in $10 \%$ normal formaldehyde and cut into sequential 5-mm blocks, from the specimen base (common carotid or proximal end) to the bifurcation, until whole the specimen was cut. Sections of each block were stained with eosin and hematoxylin. Based on the American heart association (AHA) criteria, one experienced neurosurgeon (H.K.) who was blinded to the clinical information classified atherosclerotic plaques into either type VI or not. Complicated plaques (type VI) were graded based on the following criteria: 1, organized lamellar plaque or luminal adherent thrombosis (white blood cells, red blood cells, fibrin, platelets, and lines of Zahn); 2, free red blood cells within the media or intima not associated with lumen of vessel; 3 , rupture or surface defects, or 4, macrophages containing hemosiderin.

\subsection{Statistical Analysis}

We used the StatView statistical package (StatView 5, SAS institute, Cary, NC, USA) for all statistical analysis. Spearman's rank correlation was used to analyze correlations between two factors. To compare scores of unpaired samples, Mann-Whitney U test was used. Correlations between two factors were analyzed by Spearman's rank correlation. A P value $<0.05$ was considered to indicate statistical significance. 

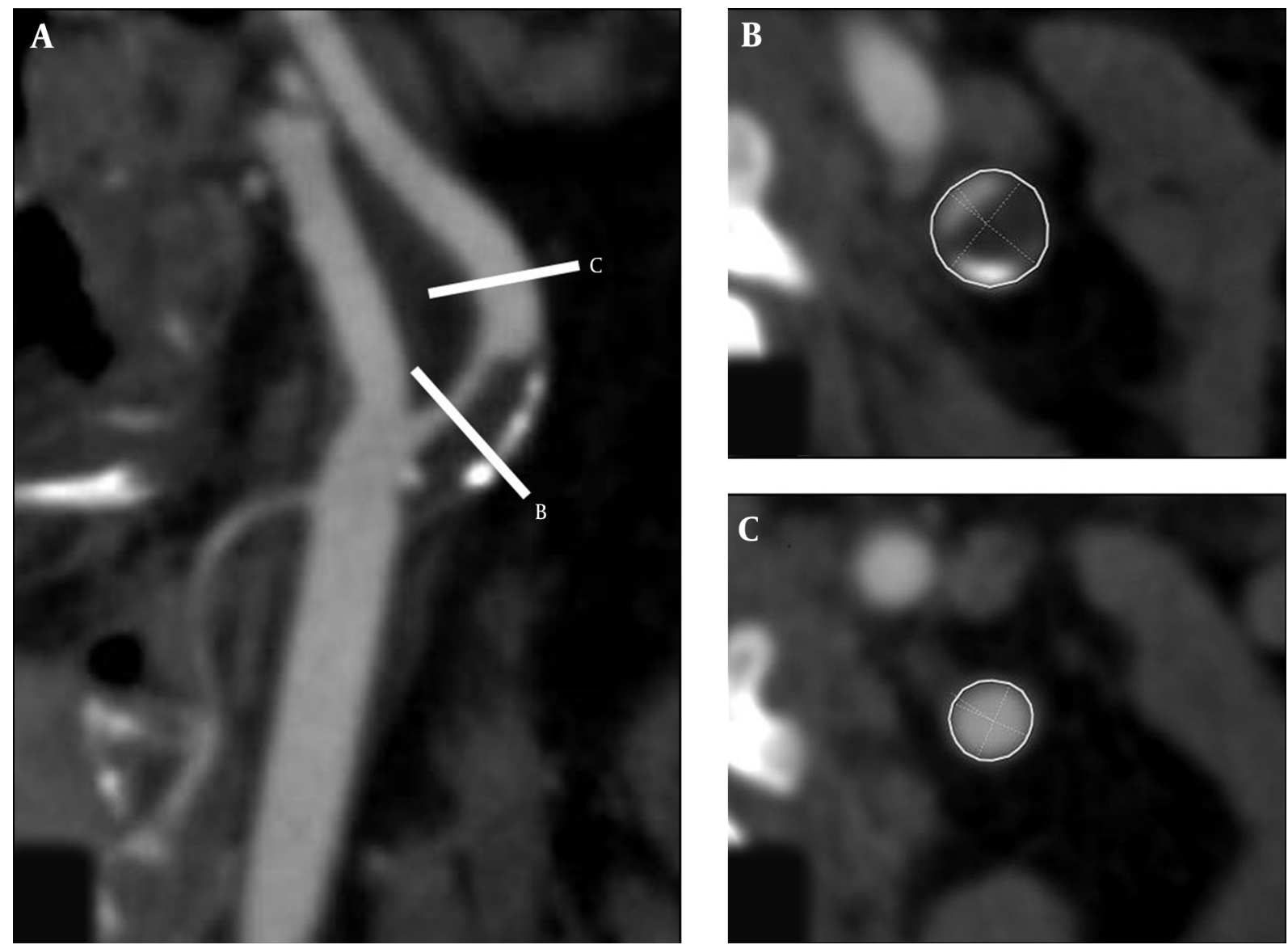

Figure 1. Multiplanar images of the carotid artery were reconstructed (A) and the region of maximum luminal narrowing was visually identified on these images and axial images (A, B). The outer vessel contour was manually traced to calculate the cross-sectional area (CSA) (B), and reference CSA was measured at the nearest distal segments without atherosclerotic plaques (C).

\section{Results}

Characteristic symptoms of the patients were transient ischemic attack, amaurosis fugax, or stroke, thought to be caused by the affected carotid artery. The definition of stroke and transient ischemic attack was based on the previously published criteria $(13,14)$. Amaurosis fugax was defined as transient acute onset of partial or complete monocular loss of vision (5).

Tables 1 and 2 show the comparison of clinical and imaging findings between 13 patients with and 16 patients without type VI lesions. There were no significant differences between the two groups regarding degree of stenosis or clinical characteristics including age, sex, symptoms, and prevalence of stroke risk factors. In contrast to the mean plaque density ( $\mathrm{P}=0.062)$, patients with type VI lesions exhibited significantly higher $\mathrm{PP}<30$ and remodeling ratios than those without type $\mathrm{VI}$ lesions $(\mathrm{P}=0.023$ and 0.002 , respectively). $\mathrm{PP}<30$ was more significantly corre- lated with the remodeling ratio than with the mean density (Figure 3; Spearman's rs $=0.76$ vs. $0.65 ; \mathrm{P}<0.0001$ and $\mathrm{P}=0.0007$, respectively). Most of the clinical parameters were not correlated with these imaging findings, except for smoking history $(\mathrm{P}=0.032)$.

\section{Discussion}

We demonstrated that the percentage of the low density plaques of less than $30 \mathrm{HU}$ calculated by histogram analysis and the remodeling ratio were correlated with the AHA type VI lesions, indicating vulnerable and complicated plaques. Of note, these complex plaques are associated with a higher incidence of cerebrovascular events (4$6)$.

Recently, the advanced imaging modalities have enabled visualizing atherosclerotic plaques. US, MRI, and CTA were non-invasive imaging modalities compared with 
Table 2. Comparison of Radiographic Parameters Between Type-VI and Non-Type-VI Groups ${ }^{\mathrm{a}}$

\begin{tabular}{lccc}
\hline Variables & All & Type-VI & Pon-Type-VI \\
\hline Remodeling ratio & $2.2 \pm 1.4$ & $3.1 \pm 1.7$ & $1.6 \pm 0.7$ \\
Mean density, HU & $56.3 \pm 32.9$ & $44.8 \pm 22.8$ & $66.4 \pm 36.7$ \\
$\mathbf{P P}<\mathbf{3 0} \%$ & $21.1 \pm 26.0$ & $29.5 \pm 26.7$ & 0.062 \\
\hline
\end{tabular}

Abbreviation: HU, Hounsfield Unit.

${ }^{\mathrm{a}}$ Values are expressed as means $\pm \mathrm{SD}$.

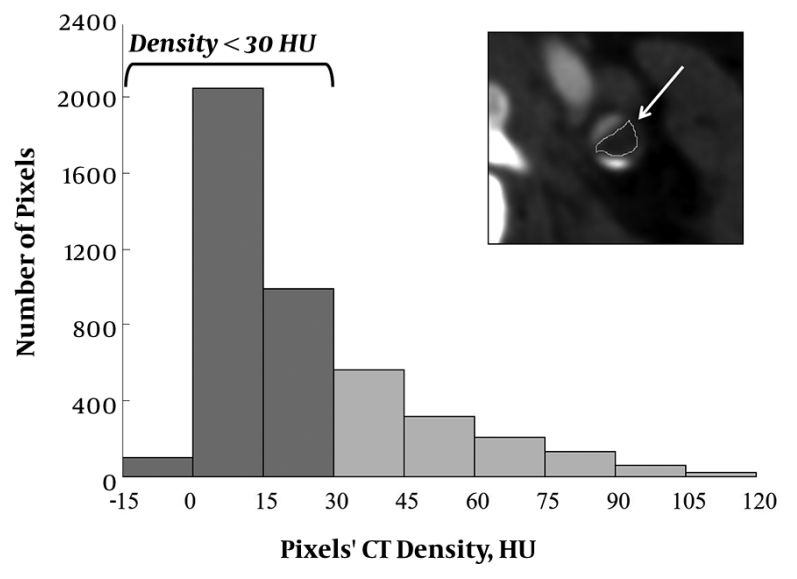

Figure 2. On the Image J program, non-calcified plaques in cross sections at the point of maximum stenosis were manually traced, and histograms of the CT value distribution were obtained. We calculated the percentage of pixels with density $<$ $30 \mathrm{HU}(\mathrm{PP}<30)$ (PP,percentage of pixels; HU,hounsfield unit ).

other modalities such as intravascular ultrasound (IVUS) (8). CTA can rapidly provide data on the whole carotid artery with high spatial resolution, making it a good candidate for routine imaging modality of carotid artery disease $(3,9)$. The assessment of percent luminal narrowing using CTA has been established in carotid artery disease in previous studies like NASCET $(3,5,8)$. Also, CTA enables visualization of plaque calcification, and morphology; smooth, irregular, or ulcerated surfaces (8). Previous studies demonstrated that diagnostic accuracy of plaque ulceration by CTA was significantly higher than by US ( 8 , 15). Recently, an additional new objective index, the remodeling ratio, has been considered useful for assessment of positive remodeling secondary to atherosclerotic plaque inflammation $(3,5,7,8)$. CT has a disadvantage that measurement of plaque density may be inferior to MRI for determining the characteristics of plaques, as described in the later section (5). MRI could detect characteristics of the vulnerable plaque with high sensitivity and specificity. However, the resolution was not high enough to detect small structures or components, and a long scan time was needed, causing partial volume effects and motion artifacts. The resolution, cardiac motion artifacts, and signal intensity were strongly influenced by hardware, venderspecific software, and scan parameters $(8,16)$. US is an easy and reproducible investigation tool and allows assessment of the degree of stenosis and morphology with high spatial resolution. However, the accuracy is operator dependent and only the cervical portion of the extracranial carotid is accurately imaged. Although the thickness of carotid intima media obtained using US has been shown to be correlated with the risk of stroke, the recent study in the general population showed no such correlation (8).

In previous studies, carotid plaque density was associated with recent ischemic neurologic events (9). It was reported that lipids, hemorrhage, and necrotic debris (called "soft tissue" by the authors) had the lowest tissue densities on CT and intraplaque hemorrhage was found in AHA type VI (4). However, the density of other tissue components (fibrosis or calcifications) and contrast agent or calcified portions in the adjacent lumen influenced the plaque densities, which affected the mean plaque densities and decreased the accuracy of plaque classification $(4,10,11)$. For the coronary atherosclerotic plaque characterization, a few studies have reported the potential role of a quantitative histogram analysis for distribution of pixels showing low density $(10,11)$. For the carotid atherosclerotic plaques, the correlation between MDCT images for measurement of lipid core area and histological sections was poor for calcified plaques in a previous study (2). In our carotid artery study, histogram analysis showed significant differences between the type VI and non-type VI groups, but mean plaque densities showed no significant differences. Also, histogram analysis showed a more significant correlation with the remodeling ratio than the analysis of mean plaque density. As the extent of expansive remodeling may indicate underlying atherosclerotic plaque vulnerability of the ICA $(3,5)$, histogram analysis may be helpful for the carotid atherosclerotic plaque evaluation on the CTA. Furthermore, the extent of the remodeling ratio correlated well with the plaque evaluation as previously reported (5).

This study had several potential limitations. First, the 
A

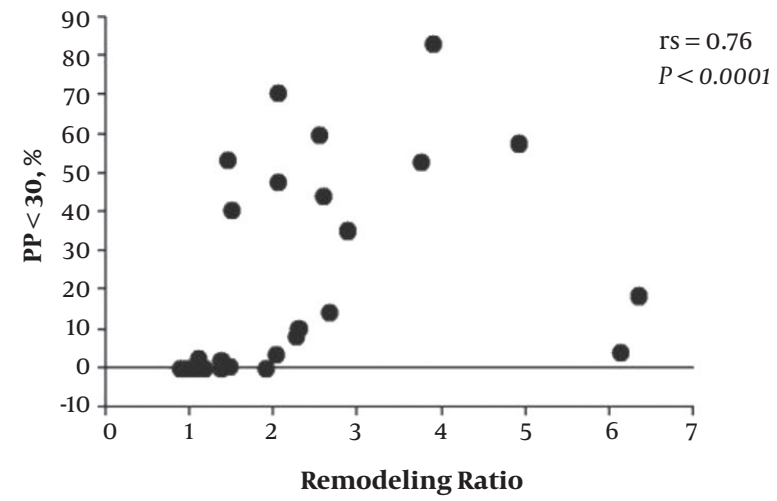

B

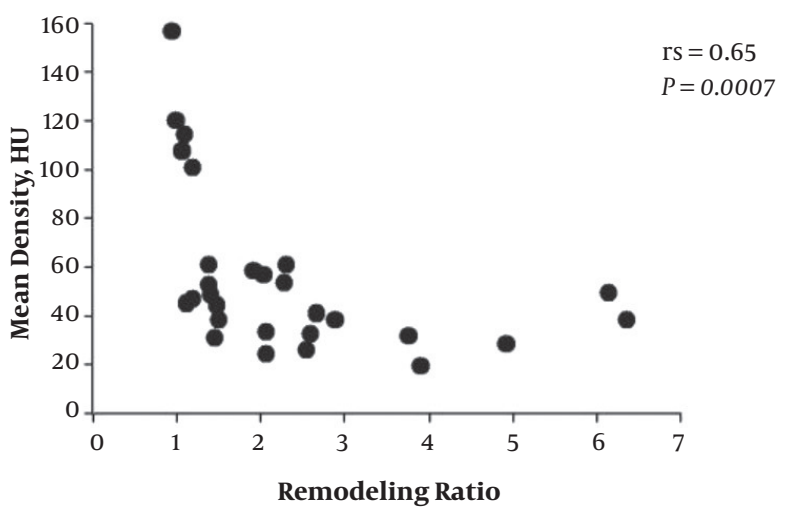

Figure 3. Correlations between $\mathrm{PP}<30$ (A) and mean density (B) with remodeling ratio were calculated. $\mathrm{PP}<30$ showed a more significant correlation with the remodeling ratio than mean density (Spearman's rs $=0.76$ vs. 0.65$)$ (PP,percentage of pixels; HU, hounsfield unit ).

presence of neurological symptoms showed no significant correlation with the remodeling ratio, PP $<30$, or AHA plaque types. As we selected patients treated by CEA, there were only four (14\%) asymptomatic patients, leading to the small sample bias. Second, as we have already mentioned in the "Patients and Methods", we selected the cutoff for low-density plaques based on the previous coronary CTA histogram study (11) using a similar scanner to ours. However, the cutoff may be less because the HU criteria of coronary plaques were reported to be significantly higher than those of carotid plaques, which are caused by more partial volume effects from lumen contrast enhancement and the surrounding tissue due to the smaller plaque size (12). In addition, the cutoff may be different according to CT vendor or number of detector rows (12). Thus, further evaluation is necessary.

In conclusion, histogram analysis of the carotid artery plaque density may be more useful for the evaluation of atherosclerosis on CTA compared with the conventional analysis of mean plaque density, and the combined analysis of histogram analysis and remodeling ratio may help to predict future stroke events.

\section{Acknowledgments}

We thank Dr. Keita Sakurai for his help in the project design and editing the manuscript.

\section{Footnotes}

Authors' Contributions: Masaki Ogawa, study concept and design, acquisition of data, analysis and interpreta- tion of data, drafting of the manuscript, statistical analysis, and study supervision; Yoshiyuki Ozawa, critical revision, technical support, and study supervision; Masahiro Muto, critical revision, and technical support; Hiroyuki Katano, analysis and interpretation of data, and technical support; Kazuo Yamada, critical revision; Toshiyasu Miura, study concept and design, critical revision; Noriyuki Matsukawa, critical revision; Yuta Shibamoto, critical revision, and study supervision.

Financial Disclosure: None.

Funding/Support: No funding and support was received.

\section{References}

1. JAMA. Endarterectomy for asymptomatic carotid artery stenosis. Executive Committee for the Asymptomatic Carotid Atherosclerosis Study. JAMA. 1995;273(18):1421-8. doi: 10.1001/jama.1995.03520420037035. [PubMed: 7723155].

2. de Weert TT, Ouhlous M, Meijering E, Zondervan PE, Hendriks JM, van Sambeek MR, et al. In vivo characterization and quantification of atherosclerotic carotid plaque components with multidetector computed tomography and histopathological correlation. Arterioscler Thromb Vasc Biol. 2006;26(10):2366-72. doi: 10.1161/01.ATV.0000240518.90124.57. [PubMed: 16902158].

3. Hardie AD, Kramer CM, Raghavan P, Baskurt E, Nandalur KR. The impact of expansive arterial remodeling on clinical presentation in carotid artery disease: a multidetector CT angiography study. AJNRAm J Neuroradiol. 2007;28(6):1067-70. doi: 10.3174/ajnr.A0508. [PubMed: 17569959].

4. Ajduk M, Pavic L, Bulimbasic S, Sarlija M, Pavic P, Patrlj L, et al. Multidetector-row computed tomography in evaluation of atherosclerotic carotid plaques complicated with intraplaque hemorrhage. Ann Vasc Surg. 2009;23(2):186-93. doi: 10.1016/j.avsg.2008.05.008. [PubMed: 18657388]. 
5. Miura T, Matsukawa N, Sakurai K, Katano H, Ueki Y, Okita K, et al Plaque vulnerability in internal carotid arteries with positive remodeling. Cerebrovasc Dis Extra. 2011;1(1):54-65. doi: 10.1159/000328645. [PubMed: 22566983].

6. Stary HC, Chandler AB, Dinsmore RE, Fuster V, Glagov S, Insull WJ, et al. A definition of advanced types of atherosclerotic lesions and a histological classification of atherosclerosis. A report from the Committee on Vascular Lesions of the Council on Arteriosclerosis, American Heart Association. Circulation. 1995;92(5):1355-74. doi: 10.1161/01.CIR.92.5.1355. [PubMed: 7648691].

7. Achenbach S, Ropers D, Hoffmann U, MacNeill B, Baum U, Pohle $\mathrm{K}$, et al. Assessment of coronary remodeling in stenotic and nonstenotic coronary atherosclerotic lesions by multidetector spiral computed tomography. J Am Coll Cardiol. 2004;43(5):842-7. doi: 10.1016/j.jacc.2003.09.053. [PubMed: 14998627].

8. Ibrahimi P, Jashari F, Nicoll R, Bajraktari G, Wester P, Henein MY. Coronary and carotid atherosclerosis: how useful is the imaging?. Atherosclerosis. 2013;231(2):323-33. doi: 10.1016/j.atherosclerosis.2013.09.035. [PubMed: 24267246].

9. Serfaty JM, Nonent M, Nighoghossian N, Rouhart F, Derex L, Rotaru $\mathrm{C}$, et al. Plaque density on CT, a potential marker of ischemic stroke. Neurology. 2006;66(1):118-20. doi: 10.1212/01.wnl.0000191391.71614.51. [PubMed: 16401860].

10. Schlett CL, Maurovich-Horvat P, Ferencik M, Alkadhi H, Stolzmann $\mathrm{P}$, Scheffel $\mathrm{H}$, et al. Histogram analysis of lipid-core plaques in coronary computed tomographic angiography: ex vivo validation against histology. Invest Radiol. 2013;48(9):646-53. doi: 10.1097/RLI.ob013e31828fdf9f. [PubMed: 23614976].
11. Marwan M, Taher MA, El Meniawy K, Awadallah H, Pflederer T, Schuhback A, et al. In vivo CT detection of lipid-rich coronary artery atherosclerotic plaques using quantitative histogram analysis: a head to head comparison with IVUS. Atherosclerosis. 2011;215(1):110-5. doi: 10.1016/j.atherosclerosis.2010.12.006. [PubMed: 21227419].

12. Kristanto W, van Ooijen PM, Jansen-van der Weide MC, Vliegenthart R, Oudkerk M. A meta analysis and hierarchical classification of HU-based atherosclerotic plaque characterization criteria. PLoS One. 2013;8(9). e73460. doi: 10.1371/journal.pone.0073460. [PubMed: 24019924].

13. Albers GW, Caplan LR, Easton JD, Fayad PB, Mohr JP, Saver JL, et al. Transient ischemic attack-proposal for a new definition. N Engl JMed. 2002;347(21):1713-6. doi: 10.1056/NEJMsb020987. [PubMed: 12444191].

14. Inzitari D, Eliasziw M, Gates P, Sharpe BL, Chan RK, Meldrum HE, et al. The causes and risk of stroke in patients with asymptomatic internalcarotid-artery stenosis. North American Symptomatic Carotid Endarterectomy Trial Collaborators. N Engl J Med. 2000;342(23):1693700. doi: 10.1056/NEJM200006083422302. [PubMed:10841871].

15. Saba L, Caddeo G, Sanfilippo R, Montisci R, Mallarini G. CT and ultrasound in the study of ulcerated carotid plaque compared with surgical results: potentialities and advantages of multidetector row CT angiography. AJNR Am J Neuroradiol. 2007;28(6):1061-6. doi: 10.3174/ajnr.A0486. [PubMed: 17569958].

16. Cai JM, Hatsukami TS, Ferguson MS, Small R, Polissar NL, Yuan C. Classification of human carotid atherosclerotic lesions with in vivo multicontrast magnetic resonance imaging. Circulation. 2002;106(11):136873. doi: 10.1161/01.CIR.0000028591.44554.F9. [PubMed: 12221054]. 